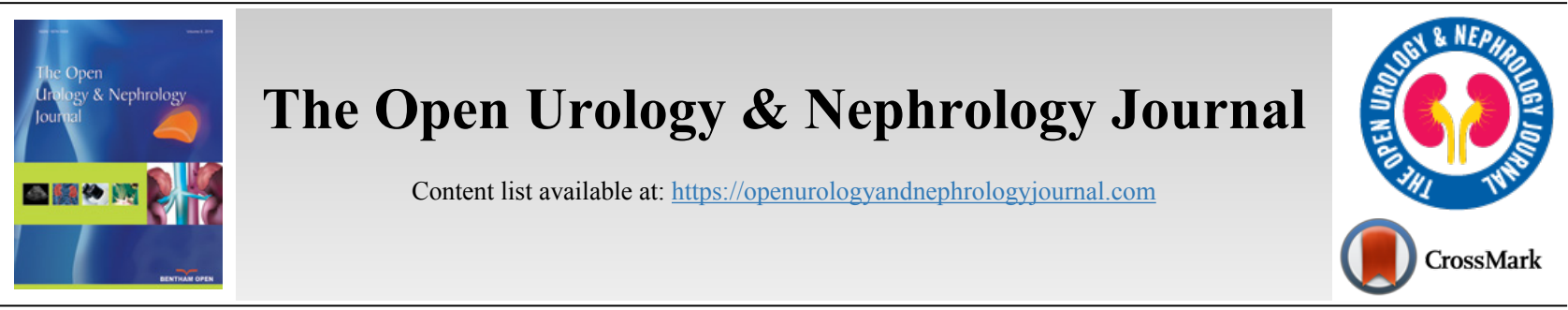

RESEARCH ARTICLE

\title{
Is Transferrin Saturation a Useful Marker of Iron Metabolism in Patients with Chronic Kidney Disease Treated with Hemodialysis?
}

Ewa Kwiatkowska ${ }^{1, *}$, Martyna Opara ${ }^{1}$, Sebastian Kwiatkowski ${ }^{2}$, Leszek Domański ${ }^{1}$, Małgorzata Marchelek-Myśliwiec ${ }^{1}$ and Kazimierz Ciechanowski ${ }^{1}$

${ }^{1}$ Clinical Department of Nephrology, Transplantology and Internal Medicine, Pomeranian Medical University, Szczecin, Poland

${ }^{2}$ Clinical Department of Obstetrics and Gynecology, Pomeranian Medical University, Szczecin, Poland

\begin{abstract}
:
Background:

According to the currently applicable KDIGO-2012 and ERBP 2013 guidelines, iron metabolism assessments for patients with Chronic Kidney Disease (CKD) are performed using such parameters as ferritin concentration and Transferrin Saturation (TSAT). Their values are to be treated as a basis on which to decide on providing iron substitution. Patients with Stage 5 CKD on maintenance hemodialysis commonly suffer from malnutrition syndrome and inflammation. One of the markers for malnutrition and inflammation is low transferrin concentration. Our study focused on establishing what percentage of patients this applied to and whether or not the transferrin saturation figure was artificially inflated in such cases.

Materials and Methods:

The study group included 66 patients with Stage 5 CKD on maintenance hemodialysis. Such data were analyzed as complete blood count, iron and ferritin concentrations, and Transferrin Saturation (TSAT). Other parameters - age, sex, time from their first hemodialysis, and the quality of their dialysis in the last six months - the $\mathrm{Kt} / \mathrm{V}$ average.

Results:

It was found that only $12 \%$ of the study group patients had their transferrin concentrations above the lower limit of normal. The TSAT value correlated negatively with transferrin concentration. Transferrin concentration correlated negatively with time from first hemodialysis or ferritin concentration, and positively with body weight. Normal transferrin concentration was only seen in patients with ferritin concentrations of up to 400 $\mu \mathrm{g} / \mathrm{L}$. The group was divided according to transferrin concentration of $<1.5 \mathrm{~g} / \mathrm{L}$ or $>1.5 \mathrm{~g} / \mathrm{L}$. These groups differed significantly in ferritin concentration and transferrin saturation. $(\mathrm{p}=0.0005$ and $\mathrm{p}=0.004$, respectively). The $1.5 \mathrm{~g} / \mathrm{L}$ transferrin concentration point divides patients with mild and medium malnutrition. It is also the minimum transferrin content necessary to achieve hemoglobin values $\geq 10 \mathrm{~g} / \mathrm{dL}$ determined using the ROC curve.

\section{Conclusion:}

Low transferrin concentrations cause abnormally high TSAT values. In most patients on maintenance hemodialysis, this marker is not useful for assessing the availability of iron for erythropoiesis.
\end{abstract}

Keywords: Transferrin saturation, Iron metabolism, Hemodialysis, Inflammation, Heomoglobin, CKD.

\begin{tabular}{|l|l|l|l|}
\hline Article History & Received: October 22, 2019 & Revised: November 28, 2019 & Accepted: November 29, 2019
\end{tabular}

\section{INTRODUCTION}

Iron is an element that many cells need in order to live. Most iron is involved where heme is synthesized. Under physiological conditions, iron insufficiency causes reduced synthesis of ferritin that is responsible for storing excessive

\footnotetext{
* Address correspondence to this author at the Clinical Department of Nephrology, Transplantology and Internal Medicine, Pomeranian Medical University, Szczecin, Poland; Tel: 509564584; Fax: (91)4661196; E-mail: ewakwiat@gmail.com
}

iron and increased synthesis of transferrin - a protein transporting iron to the spots where heme is synthesized. Unfortunately, this mechanism often malfunctions in CKD patients. According to the KDIGO-2012 and ERBP 2013 guidelines, iron metabolism is assessed and decisions on providing iron are made on the basis of ferritin concentration and transferrin saturation values. Transferrin saturation is calculated by dividing serum iron concentration by TIBC, and multiplied by 100 (Fe/TIBCx100) or Fe concentration/ transferrin concentration $\mathrm{x} 100$. Malnutrition is a common 
occurrence among CKD patients on hemodialysis, affecting $30-40 \%$ of their population $[1,2]$. There are various methods used to assess the state of nutrition, one of them being transferrin concentration assessments. It is claimed that a transferrin concentration of less than $1.0 \mathrm{~g} / \mathrm{L}$ indicates severe malnutrition, between 1.0 and $1.5 \mathrm{~g} / \mathrm{L}$ medium malnutrition, and between 1.5 and $2 \mathrm{~g} / \mathrm{L}$ mild malnutrition [3]. Another problem that this group faces is chronic inflammation, the socalled 'inflammatory syndrome', which also reduces the concentration of transferrin - a molecule referred to as a negative acute-phase protein. The inflammation experienced by patients on hemodialysis is associated with the dialysis procedure itself, as it exacerbates oxidative stress. Here, dialysis membranes stimulate cells presenting an antigen, mainly monocytes. Moreover, classic hemodialysis methods make it impossible to remove large and medium-sized particles, thus causing retention of proinflammatory molecules. Also, patients receiving hemodialysis suffer from a number of comorbidities, such as metabolic acidosis, Ca-P metabolism disorders, infections, and issues related to vascular access, causing exacerbation of both inflammation and malnutrition. The risk of cardiovascular diseases and mortality in the group of CKD patients treated with hemodialysis is 10 times higher than that in the general population, or even 1,000 times higher than in young people below 35 years old [4]. This is believed to be caused by the inflammatory process, which elevates catabolism, causes cachexia, diminishes the synthesis of many proteins - including transferrin, and leads to increased vascular calcification, thus increasing the risk of developing cardiovascular diseases. This syndrome is referred to as MIA (malnutrition, inflammation, atherosclerosis), or more adequately MICS (malnutrition-inflammation-cechexia syndrome) $[5,6]$. Few publications are available that highlight the problem of low transferrin levels in patients on hemodialysis. Transferrin is the most sensitive indicator of malnutrition and inflammation in patients on hemodialysis. This phenomenon is the cause of transferrin and its saturation ceasing to be the ideal indicator of iron status in haemodialyzed patients [7 - 11]. This doubt probably accompanies all doctors working at dialysis centres who, based on the TSAT and ferritin values, order intravenous iron supply. Our study focused on establishing what percentage of patients demonstrated hypotransferrinemia. It also attempted to determine whether or not this disorder caused abnormally high TSAT values.

\section{MATERIALS, PATIENTS AND METHODS}

\subsection{Materials and Patients}

Our analysis covered 66 patients with Stage 5 chronic kidney disease on maintenance hemodialysis performed three times a week at the Dialysis Center of the Clinical Department of Nephrology, Transplantology and Internal Diseases, Pomeranian Medical University, Szczecin, Poland. Such data were analyzed as complete blood count, iron metabolism - iron and ferritin concentrations, and Transferrin Saturation (TSAT). Other parameters taken into account were the patients' age, sex, time from their first hemodialysis, and the quality of their dialysis in the last six months - the $\mathrm{Kt} / \mathrm{V}$ average.

\subsection{Methods}

Complete blood count, iron metabolism - iron and ferritin concentrations, and Transferrin Saturation (TSAT) was determined at the Teaching Hospital's central laboratory.

\subsection{Statistical Analysis}

The data obtained in the study were analyzed. The ShapiroWilk test helped determine that the distribution was not normal. Spearman's rank correlation test was used to analyze the correlations.

\section{RESULTS}

The study group is characterized in Table 1. For these patients, the transferrin concentration median was found to be $1.58 \mathrm{~g} / \mathrm{L}$. There were 3 persons with a transferrin concentration of $\leq 1.0 \mathrm{~g} / \mathrm{L}$ (severe malnutrition), 20 with $1.0-1.5 \mathrm{~g} / \mathrm{L}$ (medium malnutrition), and 35 with $>1.5-2 \mathrm{~g} / \mathrm{L}$ (mild malnutrition). Only 8 of the study group patients were within the norm, having a transferrin concentration of $\geq 2 \mathrm{~g} / \mathrm{L}$. Also, a mere $12 \%$ of the patients on hemodialysis were within the norm for transferrin concentration. A negative correlation was found to exist between the TSAT value and transferrin concentration $(p<0.05)$ (Fig. 1). A negative correlation was discovered between transferrin concentration and time from the first hemodialysis, as well as ferritin concentration (Fig: $\mathbf{2}$ and $\mathbf{3}$ ). A positive correlation was observed between transferrin concentration and body weight (Fig. 4). The group was divided according to the degree of malnutrition. The first group consisted of patients without malnutrition and with mild malnutrition. The second group consists of patients with medium and severe malnutrition. The value separating these two groups was $1.5 \mathrm{~g} /$ $\mathrm{L}$ transferrin concentration. These groups differed significantly in ferritin concentration and transferrin saturation. $(p=0.0005$ and $p=0.004$ respectively). To determine the cut-off point of the transferrin sufficient to reach hemoglobin $\geq 10 \mathrm{~g} / \mathrm{dl}$ (recommended by KDIGO), the ROC curve was determined (Table 2). The minimum transferrin concentration needed to achieve adequate hemoglobin was $1.55 \mathrm{~g} /$ L. (Fig. 5).

\section{DISCUSSION}

As mentioned above, according to the applicable guidelines iron metabolism in Stage 5 CKD patients on hemodialysis is assessed using such parameters as ferritin concentration and transferrin saturation. As transferrin saturation is calculated as the quotient of iron level and transferrin concentration, abnormally low transferrin concentrations may inflate TSAT values. In our study, it was found that only $12 \%$ of the study group patients on hemodialysis had their transferrin concentrations within the normal range. Transferrin is a very sensitive indicator of malnutrition and inflammation. Among our patients, 5\% demonstrated concentrations characteristic of severe malnutrition, $30 \%$ of medium malnutrition, and $53 \%$ of moderate malnutrition. In Kirschbaum's study, $90 \%$ of their study population were found to demonstrate hypotransferrinemia, as well. In order to determine the transferrin cut-off 
Table 1. Characteristics of the study group (women: $37.8 \%$, men $62.12 \%$ ).

\begin{tabular}{|c|c|c|c|c|c|c|}
\hline Parameter & $\mathbf{N}$ & Average & SD & Median & MIN & MAX \\
\hline Age & 66 & 65.85 & 13.77 & 66 & 28 & 94 \\
\hline Time on dialysis (months) & 66 & 33.15 & 23.43 & 27 & 1 & 83 \\
\hline TSAT $(\%)$ & 66 & 33.55 & 15.85 & 29.5 & 9 & 102 \\
\hline Transferrin $(\mathrm{g} / \mathrm{L})$ & 66 & 1.63 & 0.36 & 1.58 & 0.75 & 2.9 \\
\hline $\begin{array}{l}\text { Ferritin } \\
(\mathrm{ng} / \mathrm{mL})\end{array}$ & 66 & 723.95 & 491.96 & 585 & 20.8 & 2100 \\
\hline
\end{tabular}

Table 2. Median values of ferritin and transferrin between groups with transferrin concentration $<1.5 \mathrm{~g} / \mathrm{l}$ and $>1.5 \mathrm{~g} / \mathrm{l}$. Statistically significant difference in Mann-Whitney $U$ test.

\begin{tabular}{|c|c|c|c|c|c|c|c|}
\hline \multicolumn{7}{|c|}{ Group } & \multirow{3}{*}{$\mathbf{p}$} \\
\hline- & \multicolumn{3}{|c|}{ I - Transferrin Concentration $<1,5 \mathrm{~g} / \mathrm{l}$} & \multicolumn{3}{|c|}{ II - Transferrin Concentration >1,5 g/l } & \\
\hline- & Median & MIN & MAX & MEDIAN & MIN & MAX & \\
\hline Ferritin ng/ml & 900 & 276 & 2000 & 421 & 20 & 2000 & 0,0005 \\
\hline $\mathrm{TSAT} \%$ & 34 & 17 & 102 & 28 & 9 & 94 & 0,004 \\
\hline Iron ug/dl & 61 & 33 & 210 & 64 & 34 & 142 & NS \\
\hline
\end{tabular}

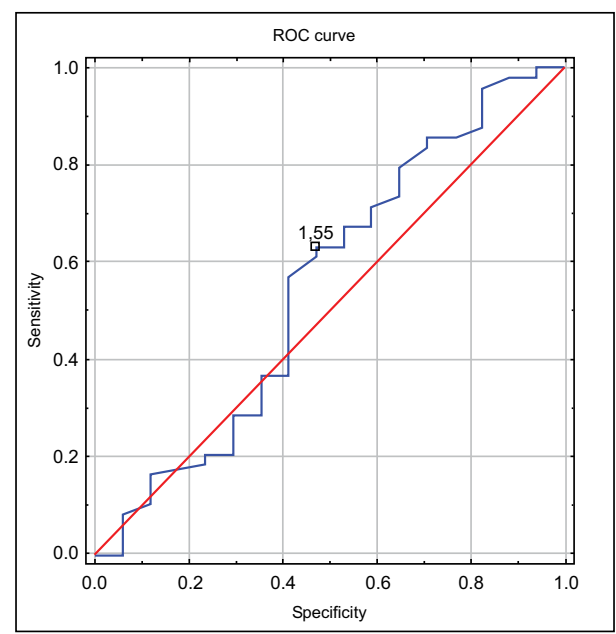

Fig. (1). The ROC curve and the transferrin cut-off point for a haemoglobin concentration greater than or equal to $10 \mathrm{~g} / \mathrm{dl}$.

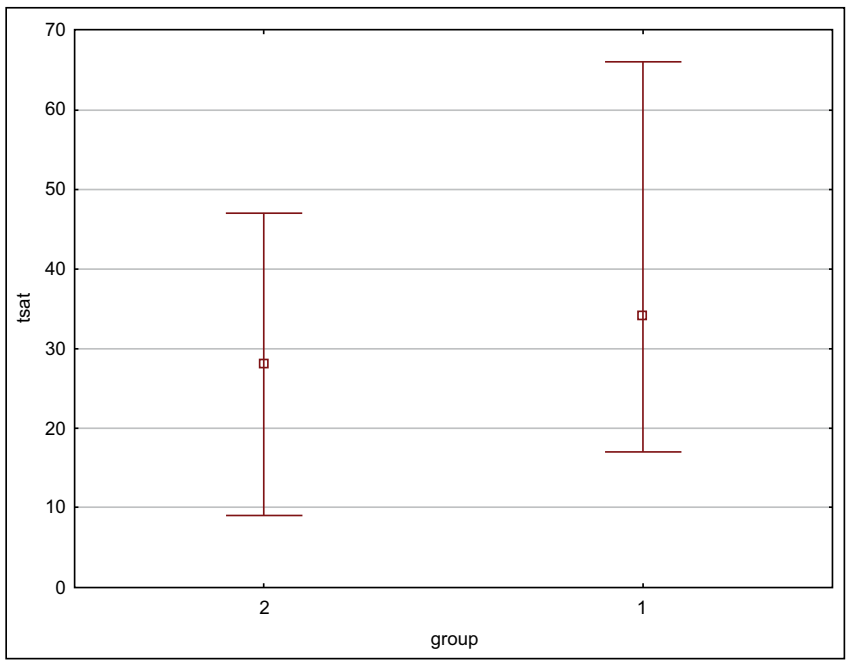

Fig. (2). The differences in TSAT percentage between group 1 (transferrin concentration $<1.5 \mathrm{~g} / \mathrm{L}$ ) and group 2 (transferrin concentration $>1.5 \mathrm{~g} / \mathrm{L}$ ). Mann-Whitney U test, $\mathrm{p}=0.004$. 


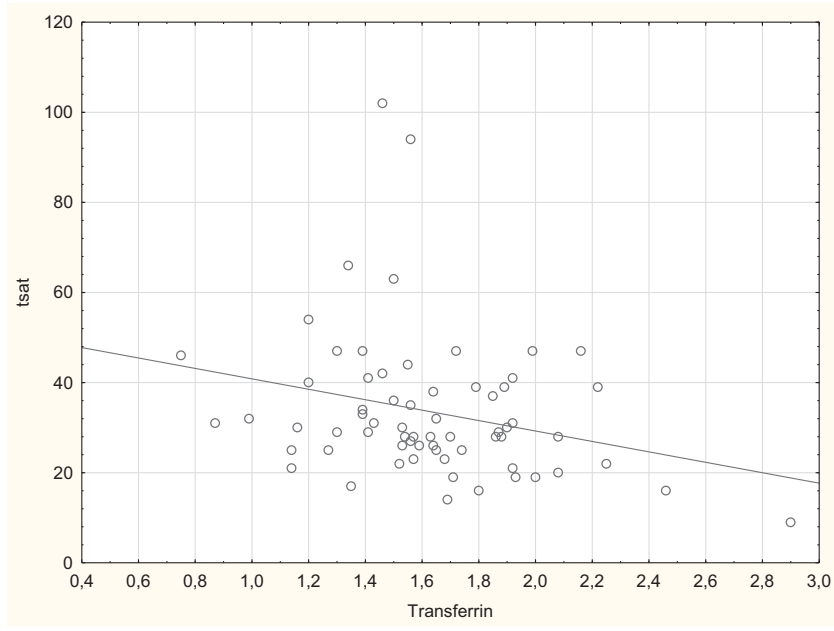

Fig. (3). Negative correlation between transferrin concentrations and TSAT $(\mathrm{p}<0.05)$.

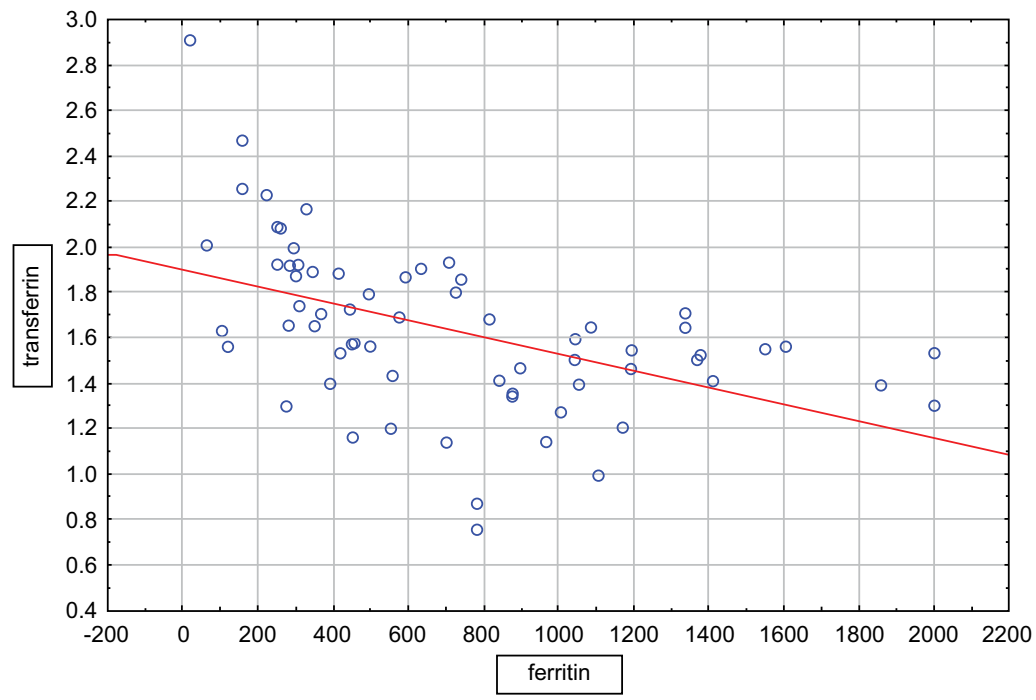

Fig. (4). Negative correlation between transferrin concentrations and ferritin concentrations $(p<0.05)$.

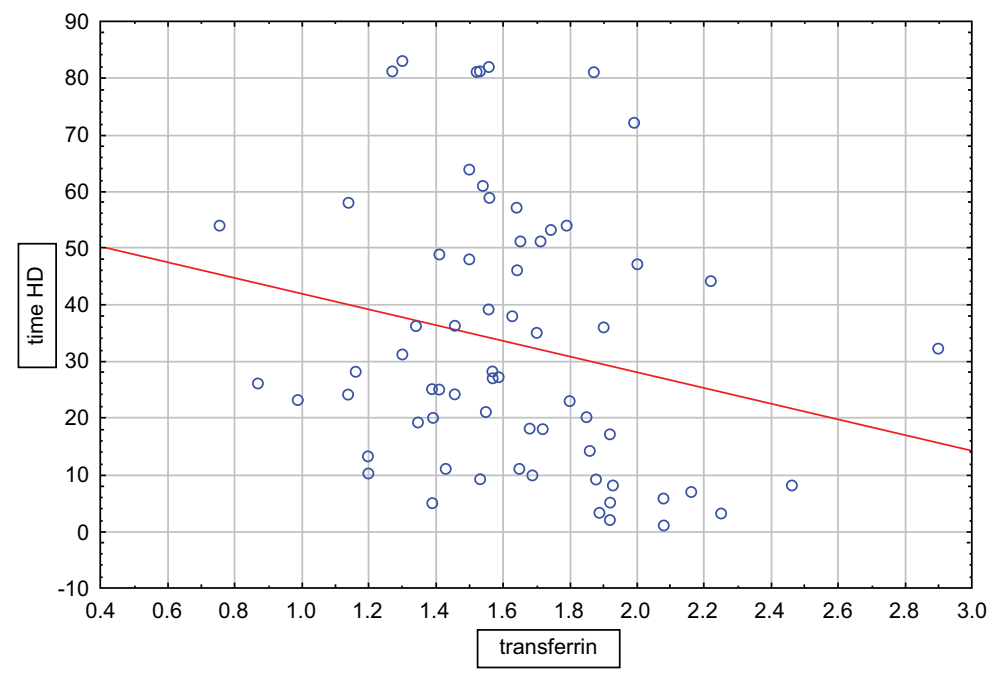

Fig. (5). Negative correlation between transferrin concentrations and time from first hemodialysis $(p<0.05)$.

point distinguishing between the ratio's values that were favorable to the hemoglobin level and those that were unfavorable to it, the ROC curve was plotted that determined the cut-off point to be at a level separating hemoglobin 
concentrations greater than or equal to $10 \mathrm{~g} / \mathrm{dL}$ from those below it. This particular hemoglobin value was assumed in this study because this is the value below which KDIGO recommends the administration of erythropoietin stimulation agents - ESA. With this hemoglobin level, the transferrin cutoff point was 1.55 (Fig. 1), which was similar to that reported in the literature dividing moderate from medium malnutrition. Hence, we used the value of $1.5 \mathrm{~g} / \mathrm{L}$ to divide our patients into two groups. In the group with a low transferrin concentration $(<1.5 \mathrm{~g} / \mathrm{L})$, we found the TSAT value to be significantly higher than in that the group with the value above $1.5 \mathrm{~g} / \mathrm{L}$ (Table 2) (Fig. 2). Low transferrin levels cause falsely high transferrin saturation results. This is confirmed by the negative correlation between TSAT and transferrin concentration (Fig. 3). This result suggests that patients with the lowest transferrin levels had the highest TSAT values. Similar results were obtained by Zadeh et al. who divided their patients treated with hemodialysis into 3 groups depending on the status of their nutrition, using the SGA (subjective global assessment) scale. It turned out that in the properly nourished group, the level of transferrin was the highest, and transferrin saturation was the lowest. However, in the significantly malnourished group, transferrin concentration was the lowest and the TSAT value was the highest [12]. The low transferrin concentration group, despite its high TSAT values, did not have a large pool of iron to be used for the synthesis of heme - 1 mol transferrin can transport $2 \mathrm{~mol}$ iron. As mentioned above, patients on hemodialysis tend to demonstrate a set of the mutually related phenomena of inflammation, malnutrition and atherosclerosis, MIC, or more adequately - inflammation, malnutrition and cachexia, where the main drive for this disorder is identified to be inflammation exacerbating catabolism and causing cachexia in the patient $[6,13,14]$. According to the available literary sources, there are numerous reasons for inflammation in hemodialyzed patients, one of which being the hemodialysis procedure itself. This correlation could be confirmed by the fact that the lowest transferrin concentration was identified in patients with the longest times from their first hemodialysis Fig. (4). It is time from the first hemodialysis that is an independent factor of malnutrition and inflammation. Ferritin is a protein whose behavior is quite contrary during inflammation - it is a so-called positive acute-phase protein. In our study, a negative correlation was found between ferritin and transferrin concentrations (Fig. 5). What is more interesting is that patients diagnosed with normal transferrin levels had ferritin concentrations not higher than $400 \mu \mathrm{g} / \mathrm{L}$ (Fig. 5). These results confirmed the presence of acute inflammation, which in hemodialyzed patients was responsible for increased ferritin levels and low transferrin concentrations. Ferritin concentrations correlated with time from the first hemodialysis, as well. A significantly higher ferritin concentration was found in the group with the transferrin concentration $<1.5 \mathrm{~g} / \mathrm{L}$ (Table 2). Proof of the influence of malnutrition on transferrin levels was found in the positive correlation between transferrin concentrations and body weight that was observed in our study group. The reverse epidemiology in the dialyzed patients group is a known fact - the 'more obese' patients are healthier. Low transferrin levels are so common among patients on dialysis that the calculated TSAT is in most cases incorrect. Low values of transferrin cause high TSAT, which is a contraindication to iron substitution. In the group with transferrin deficiency, the ferritin level is also significantly higher, which means that these patients may not receive the necessary substitution despite their actual iron deficiency.

\section{CONCLUSION}

Low transferrin concentrations are found in most patients on hemodialysis, which is why we should not use the transferrin saturation indicator for this group, as it is artificially inflated. Transferrin is a negative marker for malnutrition and inflammation, and is reduced as the time from the first hemodialysis increases. Also, it is reduced as ferritin concentration grows, and is higher in patients with larger body weights.

\section{ETHICS APPROVAL AND CONSENT TO PARTICIPATE}

Not applicable.

\section{HUMAN AND ANIMAL RIGHTS}

Not applicable.

\section{CONSENT FOR PUBLICATION}

Informed consent was obtained from all individual participants included in the study.

\section{AVAILABILITY OF DATA AND MATERIALS}

The data supporting the findings of the article is available in the RepOD at. RepOD. http://dx.doi.org/10.18150/repod. 6027166 .

\section{FUNDING}

None.

\section{CONFLICT OF INTEREST}

The authors declare no conflict of interest, financial or otherwise.

\section{ACKNOWLEDGEMENTS}

Declared none.

\section{REFERENCES}

[1] Cianciaruso B, Brunori G, Kopple JD, et al. Cross-sectional comparison of malnutrition in continuous ambulatory peritoneal dialysis and hemodialysis patient. Am K Kidney Dis 1995; 25: 475-86. [http://dx.doi.org/10.1016/0272-6386(95)90494-8]

[2] Kuciel G, Łysiak-Szydłowska W. Malnutrition and nutrition assessment. Anestezjol Intens Ter 2001; 1: 29-33.

[3] Ikizler TA, Hakim RM. Nutrition in end-stage renal disease. Kidney Int 1996; 50(2): 343-57.

[http://dx.doi.org/10.1038/ki.1996.323] [PMID: 8840260]

[4] Levey AS, Beto JA, Coronado BE, et al. Controlling the epidemic of cardiovascular disease in chronic renal disease: What do we know? What do we need to learn? Where do we go from here? Am J Kidney Dis 1998; 32: 5-13.

[http://dx.doi.org/10.1053/ajkd.1998.v32.pm9820463]

[5] Stenvinekl P, Heimburger O, Lidholm B, et al. Are there two types of malnutrition in chronic reanl failure? Evidence for relationschip between malnutrition, inflammation and atherosclerosis (MIA syndrome). Nephrol Dial Transplant ; 200(15): 953-60.

[6] Kalantar-Zadeh K. Recent advances in understanding the malnutrition- 
inflammation-cachexia syndrome in chronic kidney disease patients: What is next? Semin Dial 2005; 18(5): 365-9.

[http://dx.doi.org/10.1111/j.1525-139X.2005.00074.x] [PMID: 16191172]

[7] Gaweda AE. Markers of iron status in chronic kidney disease. Hemodial Int 2017; 21(Suppl. 1): S21-7. [http://dx.doi.org/10.1111/hdi.12556] [PMID: 28328097]

[8] Van Wyck DB, Alcorn H Jr, Gupta R. Analytical and biological variation in measures of anemia and iron status in patients treated with maintenance hemodialysis. Am J Kidney Dis 2010; 56(3): 540-6. [http://dx.doi.org/10.1053/j.ajkd.2010.05.009] [PMID: 20638166]

[9] Wish JB. Assessing iron status: Beyond serum ferritin and transferrin saturation. Clin J Am Soc Nephrol 2006; 1(Suppl. 1): S4-8. [http://dx.doi.org/10.2215/CJN.01490506] [PMID: 17699374]

[10] Fishbane S, Kowalski EA, Imbriano LJ, Maesaka JK. The evaluation of iron status in hemodialysis patients. J Am Soc Nephrol 1996; 7(12): 2654-7.
[PMID: 8989744]

11] Reddy GC, Devaki R, Rao P. Iron Indices in Patients with functional anemia in chronic kidney disease. EJIFCC 2013; 24(3): 129-36. [PMID: 27683448]

12] Kalanter-Zadeh K, Kleiner M, Dunne E, et al. Total iron-binding capacity - estimated transferrin correlates with the nutritional subjective global assessment in hemodialysys patients. Am J Kidney Dis 1988; 31: 263-72.

[http://dx.doi.org/10.1053/ajkd.1998.v31.pm9469497]

[13] Stenvinkel P, Heimbürger O, Lindholm B. Wasting, but not malnutrition, predicts cardiovascular mortality in end-stage renal disease. Nephrol Dial Transplant 2004; 19(9): 2181-3.

[http://dx.doi.org/10.1093/ndt/gfh296] [PMID: 15238625]

[14] Jofré R, Rodriguez-Benitez P, López-Gómez JM, Pérez-Garcia R. Inflammatory syndrome in patients on hemodialysis. J Am Soc Nephrol 2006; 17(12)(Suppl. 3): S274-80.

[http://dx.doi.org/10.1681/ASN.2006080926] [PMID: 17130274]

(C) 2019 Kwiatkowska et al.

This is an open access article distributed under the terms of the Creative Commons Attribution 4.0 International Public License (CC-BY 4.0), a copy of which is available at: https://creativecommons.org/licenses/by/4.0/legalcode. This license permits unrestricted use, distribution, and reproduction in any medium, provided the original author and source are credited. 